\title{
Human-Driven Design: A Human-Driven Approach to the Design of Technology
}

\author{
Marketta Niemelä, Veikko Ikonen, Jaana Leikas, Kristiina Kantola, Minna Kulju, \\ Antti Tammela, and Mari Ylikauppila
}

VTT Technical Research Centre of Finland, Tampere, Finland

\begin{abstract}
In the midst of the many large-scale societal and technological transformations, there is a need for design approaches that respect human values and needs and are able to integrate multiple perspectives into technology design in order to work for outcomes that are interesting, feasible and sustainable in all senses of the term. For this purpose, we discuss a possible approach to the design of technology that is driven by human and social values, is collaborative in nature and reflective in terms of responsibility and ethics in the design. We call this approach 'Human-Driven Design' and argue that it is needed especially when designing for enabling and emerging information and communication technologies. A human-driven design approach should focus on the early phases of design, be strongly future-oriented and aim to contribute to innovation for a sustainable society and better quality of life in the future.
\end{abstract}

Keywords: human-driven design, human-technology interaction, human values and needs, emerging ICTs.

\section{Introduction}

It has become increasingly important in the field of human-computer interaction (HCI) to understand the demands and opportunities that societal changes bring to technology development and interaction design. The grand challenges, such as climate change, an ageing population and the economic crisis, demand participation by and contributions from everyone, and HCI, combined with technology development and its vigorous expansion into our everyday lives, plays a key role. To deal with this, the intersection(s) between people, technology and design in HCI need(s) to be rethought and the human-centred practices need more human values put into them (e.g. $[1,2]$; see also [3]). Human values and needs should be better integrated into design to obtain solutions that are sustainable in all senses of the term.

There are a variety of approaches or frameworks that specifically emphasise a human perspective in design, such as participatory design and value-sensitive design, to mention a couple. In this paper, we review five of these frameworks and formulate this understanding for a more generic human-driven approach (or strategy) to design Human-Driven Design (HDD). As shown later in the text, this approach is based on emphasising human and social values, seeking true collaboration and empowerment with various stakeholders in the design process, and being reflective in terms of responsibility and ethics in design. 
As a term, human-driven design has been introduced before by Braund and Schwittay [4] (we present their perspective in Section 2.2) and Ikonen [5]. Ikonen presents HDD as an approach to the design of future smart environments and emerging ICT and describes HDD as an "approach which broadens the perspective from focused product or service development process model to the more holistic design perspective". Ikonen also describes stakeholder-based design that "furthermore broadens the scope and role of involved participant groups in the actual design process". Ikonen further calls for ethical assessments that should continue through the design process. Ikonen calls this resulting combination empowering design.

Based on our review, we agree that HDD should integrate the three perspectives endorsed in [5]: it should be holistic, strive for collaboration with different stakeholder groups and be ethically reflective. The present paper elaborates on our understanding of what human-driven design is in more detail for each perspective.

We characterise HDD as follows:

1. HDD takes a human and social view of users of technology as individuals and members of human social groups, such as family, organisation or community, and as consumers. The starting point of the design is the human being and her or his needs, goals and desires. The design is based on a deep understanding of the users' values and circumstances.

2. HDD is participatory in terms of promoting collaborative and co-design methods to empower users and other stakeholders in design and to ensure successful design outcomes.

3. HDD is responsible in terms of being aware of human, societal and ethical values related to a particular design and reflecting them in the design in order to make the technology support the wellbeing and activities of people as well as sustainability (social, environmental and economic).

Similarly to [5], we find the human-driven approach especially relevant in the context of emerging technologies and their future impacts. As technologies embed and intertwine in our everyday lives, they will also shape it and society: technology is increasingly something "we live with, not simply something we use" [1]. In some sense, to design technology is to design our future lives. Therefore, the design of technology should be formulated with an insight into human and societal phenomena and problems, sustainable development and business demands.

From this perspective, the design target of HDD would not just be human-computer interaction but broader Human-Technology Interaction (HTI) that emphasises the importance of research and design considerations that extend beyond the 'normal' usercomputer combination. HTI addresses the interaction between users, technology and the physical and social environment [6]. In HTI, there is particular interest in the way technologies mediate the interaction between human actors and the environment, and in how to develop artefacts for the human-environment interaction. The holistic perspective of HTI is important when the purpose is to design solutions for human or societal problems (for example, to improve quality of life; see, e.g., [7]) and not just solutions for and within a certain technology. In practice, our current thinking about HDD is mostly based on developing ICT and emerging ICT (e.g. applications and services based on ambient intelligence). Therefore, with 'design' or 'technology design' we mainly refer to the 
design of the interaction between humans and computer systems, especially emerging information and communication technologies and systems.

With this paper, we hope to open discussions with other HCI/HTI researchers or designers who feel they could benefit from having a more holistic, human-driven approach to apply to design.

We proceed as follows. First, we position HDD in the field of HCI/HTI research by discussing some influential frameworks and research that form its basis: HumanCentred Design, Participatory Design, Value Sensitive Design, Human-Driven Design and Research, and Life-Based Design. Next, we elaborate on HDD by examining its three principles. Finally, we discuss what is still needed to develop HDD as a useful alternative to designing emerging (information) technologies.

\section{Related Frameworks}

\subsection{Human-Centred Design}

Human-Centred Design (HCD) is the prevailing general human-perspective framework for the design of interactive products, services and systems [8]. The aim of HCD is to make systems usable and useful by focusing on the users, their needs and requirements. The ISO standard for HCD defines both 'usability' and 'usefulness' broadly: for instance, usability may include aspects of use that are typically associated with user experience, such as emotional pleasure. Applying HCD to design should show, for example, increased productivity, improved ease of use, and user experience or reduced discomfort and stress.

HCD emphasises understanding the users and contexts of direct use, and it recommends the involvement of users throughout the design and development, including long-term monitoring of the product. HCD also addresses the impacts on stakeholders other than direct users.

The HCD standard also includes a perspective on sustainability [8]. Economic sustainability is supported as HCD provides cost-effective solutions and reduces the risk that the designed product will be wasteful or rejected. As the application of HCD results in products that are better for the health, well-being and involvement of their users, it supports social sustainability. HCD indirectly contributes to environmental sustainability as it encourages consideration of the long-term implications of systems.

The HCD framework provides several important principles for HDD: the human perspective itself, as well as a broad understanding of the users and their context, and the stakeholders. However, HDD should apply ethical and responsibility reflections more prominently in design. One reason for this is that emerging technologies often raise ethical considerations. For example, in the case of ubiquitous computing, ethical concerns have been raised, especially from the point of view of privacy, confidentiality, integrity, availability, trust, control and autonomy (e.g. [9]). Another issue that we have found to be underemphasised in HCD is justification and meaning of technology to users. 


\subsection{Participatory Design}

Participatory Design (PD) is another well-established human-centred approach that emphasises active involvement by the users and all the stakeholders in design. PD involves or proposes: 1) mutual learning between users and designers about their respective fields, 2) the use of tools in the design process that are familiar to the users, 3 ) envisionment of future work situations to allow the users to experience how emerging technologies may affect the work practice, and 4) the importance of starting the design process in practice for the users [10] (referred to in [11]).

The original purpose of PD was emancipatory, to empower workers by involving them in the development of new systems for the workplace. Since then, PD has been applied to the development of systems outside the work context, such as leisure activities, entertainment and social service provision [11]. As the design focus of PD has evolved, the understanding of what PD is may also have changed. Nowadays, PD can be understood as a "practice of collective creativity" and it is rather called co-design or co-creation [12] (p. 7).

A major impact of participatory design or co-design concerns the roles of the stakeholders, namely the user, researcher and designer [12]. The users become codesigners, and active and creative participants, to some extent at least. The researcher changes from a translator of information between the users and the designer to a facilitator who encourages and allows people's expressions of creativity at all levels. And, even if the user is a co-designer, there is a specific place for the professional designer, whose expertise on design thinking and contextual issues (technologies, production processes, business) is needed even more than before in order to solve global, systemic problems.

Sanders and Stappers [12] note that today, co-design is very market-oriented and even an elite project with the focus on the "lead users". The original idea of PD, of emancipation and empowering the user to influence her or his living and working environment, has been somewhat blurred under such design ideas as open innovation or user-driven innovation with direct business aims. Co-design of products and services is a positive trend in production and business, but PD and empowering the user also have greater potential to help people to understand large-scale sociotechnological changes and their expected impacts, and, in particular, to influence them.

\subsection{Value Sensitive Design}

Value Sensitive Design (VSD) is a widely known method for ethical design of technology $[13,14]$. VSD provides a framework into which to integrate human values with ethical imports to design systematically and from different perspectives. 'Value' is broadly defined as something that a person or group considers important in life [14]. However, VSD states that certain values are universally held, although they may manifest themselves in various forms in different cultures and times.

The iterative methodology provided by VSD includes conceptual, empirical and technical research and evaluation work. The process starts with the conceptual phase, 
the purpose of which is to identify the design stakeholders and their values, in addition to those stakeholders that interact with the designed technology directly or indirectly. The central values are also conceptually defined; for instance, what is meant by trust, on what does trust depend and how can trust be operationalised as concretely as possible? Next, the empirical phase concentrates on identifying and evaluating how the central values are implemented in practice in activities and interactions with people and systems. Finally, in the technical phase, the properties and background mechanisms of technology are studied to understand how they either support or hinder the implementation of certain values. This phase also includes anticipative, technical design that supports the integration of the central values into the design results.

Value lists can be used to support and guide design in a heuristic manner [14]. Typical values include human wellbeing, freedom of bias with regard to technical or social issues, accountability for one's own actions and thoughtfulness. Several of the values named by Friedman and her colleagues relate to privacy and data handling.

The original idea of VSD that certain values are universal has raised discussion among researchers. For instance, Borning and Muller [15] argue that this statement impedes adopting VSD by researchers and designers. They suggest that VSD should accept the idea of pluralism, i.e. the simultaneous existence of several alternative values. VSD should endeavour to contextualise the expressions of values in different ways, for instance when using value lists as a tool or writing research reports.

VSD may also be difficult to apply in practice because the methods of VSD do not provide concrete guidance for how to integrate ethics in design. Huldtgren [16] asks whether a "design team need[s] to include a social scientist or someone trained in the [VSD] methods above to be able to carry out VSD" (p. 87). Expertise on ethics is not always easily acquired in industry, and the role of "value advocates" may be difficult [17]. To support overcoming this problem, Huldtgren helpfully provides some practical questions to be considered when using VSD in practice, such as "Which values are important in a given design case?", "Whose values are they?" and "Which methods are suited to discover, elicit and define values?" [16] (p. 88).

\subsection{Human-Driven Design and Research}

The framework for Human-Driven Design and Research (HDDR) [4] has been formulated in the context of ICT development projects for developing countries. Here, one important question is how to balance the pull exerted by local wants and aspirations with the push from global institutional and technological forces.

Braund and Schwittay [4] argue for focusing on real human needs when using ICT and point out that there must be "room for possibility that for certain groups ICT is not (at least at the moment) an appropriate solution to their problems". The response by HDDR to this call is that it is being driven by in-depth knowledge about local conditions gained from long-term human-centred research and a participatory community design process. HDDR recognises that the success of ICT development needs understanding and action in all four dimensions: local practices, a participatory design process, sociocultural contexts and political conditions. For instance, attempts to involve the community in development projects (participatory action) may fail because the necessary 
preconditions for the involvement to take place are not created (e.g., local practices, political conditions).

HDDR sees the importance of technology in improving the conditions of human life but simultaneously recognises that technology can become just "another tool of political, economic and social exclusion". To prevent this, HDDR promotes multidimensional collaboration and listening to all the stakeholders in the development.

HDDR and HDD seem to grow from the same ground; only the context of design is different. In developing countries, HDDR probably needs to deal with much stronger conflicts between Western economic culture, and local needs and culture. Otherwise, both share the concern of how to make local people heard and empowered by the design process and result. Furthermore, both focus on the question of how to integrate ICT into people's lives so that it makes a meaningful difference.

\subsection{Life-Based Design}

The responsibility of design, the justification for technology and the question of 'good technology' are essential aspects of HDD thinking for which we draw theoretical strength from Life-Based Design (LBD). This is a multi-dimensional and holistic approach to the design of technology and the information society developed in [7] and $[18,19,20]$. LBD calls for understanding the true value of technology as a means to the ultimate target of improving the quality of human life: what technology could really offer people and in what forms and on what terms it would be welcomed and adopted.

LBD emphasises a holistic understanding of human life as the foundation of the design: life is conceptualised and analysed from different perspectives (biological, psychological, socio-cultural) as Forms of Life that technology should support and advance in order to enhance quality of life. Forms of Life analysis paves the way for concept design (e.g. by practicing HCD).

Concepts are further exposed to Fit-for-Life analysis that examines the ethicality of the design solutions and the benefit and meaningfulness that users can receive from the solutions that are developed and how these can be improved for better quality of life. Finally, the phase of innovation design defines a procedure for creating usage cultures and exporting the outcome for general use.

In short, LBD is an approach that involves the concepts of human life sciences through the span of the design of technology and the information society in general: from (e.g. ethically reflected) ideas to (collaboratively designed) concepts to technology use and its impacts on quality of life and usage cultures [7]. For HDD, LBD offers the ground framework with holistic research paradigms for organising design thinking and carrying out research and design activities.

\subsection{Summary}

We have used the five frameworks described above - Human-Centred Design, Participatory Design, Value Sensitive Design, Human-Driven Design and Research, and Life-Based Design - to build our understanding of what Human-Driven Design is, in 
particular, what the central principles of the approach are (Table 1). First of all, HDD takes a perspective of the human and her or his values, the living and working environment, and other conditions of human life as a starting point in the design. Second, the users and other stakeholders are not just identified and recognised in the design process but involved as active participants in the design to the extent that the presupposed roles of the researcher and designer change, and all the participants are empowered. Finally, HDD goes beyond designing 'just' a product, service or system. HDD includes a larger, perhaps societal, point of view. We call this characteristic 'responsibility'. It expresses itself in different ways as promoting social and economic sustainability, and ethical and moral values.

Table 1. Principles of HDD design based on the five influential frameworks

\begin{tabular}{|l|l|l|l|}
\hline \multirow{2}{*}{$\begin{array}{l}\text { Design } \\
\text { approach }\end{array}$} & \multicolumn{3}{|c|}{ Principles of human-driven approach } \\
\cline { 2 - 4 } & $\begin{array}{l}\text { Human and social } \\
\text { view }\end{array}$ & Participation & Responsibility \\
\hline $\begin{array}{l}\text { Human- } \\
\text { Dentred }\end{array}$ & $\begin{array}{l}\text { Human perspective on } \\
\text { iterative design }\end{array}$ & $\begin{array}{l}\text { Participatory approach } \\
\text { to involve users and } \\
\text { other stakeholders in } \\
\text { the design }\end{array}$ & $\begin{array}{l}\text { Direct support for } \\
\text { social and economic } \\
\text { sustainability }\end{array}$ \\
\hline $\begin{array}{l}\text { Participatory } \\
\text { Design }\end{array}$ & $\begin{array}{l}\text { Empowering users (as } \\
\text { workers, co-creators } \\
\text { and co-designers) }\end{array}$ & $\begin{array}{l}\text { Involving users } \\
\text { throughout all the } \\
\text { phases of the design }\end{array}$ & $\begin{array}{l}\text { Advances democracy } \\
\text { and equality between } \\
\text { people }\end{array}$ \\
\hline $\begin{array}{l}\text { Value Sensi- } \\
\text { tive Design }\end{array}$ & $\begin{array}{l}\text { Integrates human } \\
\text { values into design }\end{array}$ & $\begin{array}{l}\text { All stakeholders' } \\
\text { values are identified } \\
\text { and recognised }\end{array}$ & $\begin{array}{l}\text { Promotes values with } \\
\text { ethical and moral } \\
\text { import }\end{array}$ \\
\hline $\begin{array}{l}\text { Human- } \\
\text { Driven De- } \\
\text { sign and } \\
\text { Research }\end{array}$ & $\begin{array}{l}\text { ICT as a tool to make } \\
\text { a meaningful differ- } \\
\text { ence }\end{array}$ & $\begin{array}{l}\text { Emphasis on local } \\
\text { practices and empow- } \\
\text { ering people }\end{array}$ & $\begin{array}{l}\text { Awareness of conflicts } \\
\text { of interests between } \\
\text { stakeholders in devel- } \\
\text { opment }\end{array}$ \\
\hline $\begin{array}{l}\text { Life-Based } \\
\text { Design }\end{array}$ & $\begin{array}{l}\text { Justification for tech- } \\
\text { nology: to improve } \\
\text { quality of life. Under- } \\
\text { standing of human life } \\
\text { as the foundation of } \\
\text { the design }\end{array}$ & $\begin{array}{l}\text { Concept design is } \\
\text { based on the analysis } \\
\text { of a form of life, sup- } \\
\text { plemented by ideation, } \\
\text { reflection and elabora- } \\
\text { tion of solutions with } \\
\text { users }\end{array}$ & $\begin{array}{l}\text { Reflections of real } \\
\text { human needs. Analysis } \\
\text { of fit for life and long- } \\
\text { term impacts. Value } \\
\text { and ethicality of de- } \\
\text { sign solutions }\end{array}$ \\
\hline
\end{tabular}

\section{Reflecting Human-Driven Design}

We now elaborate on the three principles of HDD: the human and social perspective on design, participation and responsibility. We also illustrate these principles with some practical experiences and methods. The purpose of this chapter is to help other practitioners in the HCI/HTI field to recognise and reflect on how they can apply 
(parts of) HDD to their own work, either in the design of emerging technologies for the future society or in other contexts.

\subsection{Human and Social View}

HDD promotes a human perspective on technology design in a profound way: technology should make a meaningful difference, and the justification for technology should be to improve quality of life. A true ICT society should be examined and developed through human dimensions and the concept of quality of life. In current HTI design, usage situations are too often the main area of concentration, while ignoring the real needs that arise out of people's needs. Access to and usability of technology do not yet imply use, however, and use does not necessarily imply meaningful use. A successful solution creation process needs to look at technology as one possible enabler and not as the default tool [21]. HDD thus applies methods to understanding social and cultural settings for the development of emerging technologies and how to assess the meaningfulness of technology for the user. These refer to values, expectations, beliefs and practices that also influence the way individuals interpret the world and technology. They have significant influence on the individual's involvement in technology and on the way a community is able to make use of technology (see, e.g., [22]).

The Fit-for-Life design [19], for example, is a method (under development) to test the meaningfulness of a technology concept - its fit in the lives of future users to ensure that it will enhance the quality of life. The method should be applied after the concept design phase, before proceeding to the implementation. The Fit-for-Life analysis refers to re-checking the concept against the human requirements defined in earlier design phases (e.g., through the holistic Form-of-Life analysis). At the same time, the requirements should be reflected for the needs of the implementation phase. If there is a mismatch between the concept and the requirements, the concept should be refined.

\subsection{Participation}

HDD seeks users' active, co-creative involvement throughout the design span. The other stakeholders such as technology developers and service providers are of equal importance. HDD could be described in the words of Marc Steen [3]: with all stakeholders, HDD aims to "engage in a dialogue in which we can learn from each other, and jointly explore and articulate ideas about needs we can try to solve", and moreover "create a setting in which different people contribute their skills and ideas so that we can jointly explore, envision and evaluate ideas, concepts and new products" (p. 15).

To illustrate the participatory aspect of HDD, we present four example co-design approaches developed or applied by us as a HCI/HTI research team. Our aim has been to develop human-driven methodology in which participation in design occurs close to the real use context and as intertwined with everyday life, similarly to Ikonen et al. [23]. Human-driven methods set a low threshold for participation but aim to bring value for all stakeholders. The methods seek to provide optional ways to contribute 
and encourage creativity and informal interaction, and enable agile gathering and analysing of data as well as support for iterative development.

IHME - an Open, Public Showroom and Co-design Environment. In 2010, we designed and implemented an open, public technology showroom and co-design environment in small commercial premises in a shopping centre [24]. The purpose of the showroom was to demonstrate an open, low-threshold co-design environment in which different stakeholders of technology design could meet and learn from each other. The place opened its doors to attract shoppers and other passers-by to pop into the premises to familiarise themselves with the latest advancements in ICT (namely, 3D television, augmented reality, virtual travelling, etc.) and to participate in user studies. Two employees worked at the premises full time to show visitors around, interview them and organise questionnaire studies. The showroom was open for two months in the summer of 2010, during which time it had approximately 2500 visitors. The feedback from the visitors was positive: the environment was considered fun, informative, interesting and easy to approach.

Owela - A Social Media Platform for Co-design. Another method that we have developed and systematically apply for easy involvement in design is the online platform 'Owela' (Open Web Lab), built on social media-type interaction, which thus enables user participation regardless of time and place [25]. This platform provides tools and methods (discussion, polls, voting, online chatting, etc.) for understanding users' needs and experiences as well as innovating and designing new products and services together. The platform enables different levels of participation based on the users' own interests and provides scoring tools to reward and motivate the participants. The advantage of online co-design is that all the developers have real time access to user feedback without intermediates. The developers have an easy option to ask questions directly to the users. This supports reciprocal understanding between the users and developers.

Visual IHME - A Visual Online Platform for Co-designing Places. The aforementioned method, Owela, has been further developed towards a more visual co-design platform that utilises realistic images of places. This platform provides a photo-based, interactive spherical panorama environment with a set of easy-to-use co-design interaction tools such as discussion boards, questionnaires and polls on the screen (on the panorama image). All user-created content can be pinned to specific spatial spots on the image. The platform can be used for HDD purposes to study and co-design indoor and outdoor places, i.e. for understanding, co-creating and sharing meanings bound to or growing from specific locations. In this sense, the platform can be used, for instance, to promote sustainable behaviour [26, 27].

Living Lab. Other 'human-driven' methods are those in which participation is embedded in the routines and practices of the user in a long-term manner - so-called Living Labs. Our most recent Living Lab case focused on the development of future postal services. The Living Lab was based on a small village, and the participant 
households (approximately half of the village) committed to the iterative co-design and development process for several months. A variety of methods from household and individual interviews and questionnaires to co-design sessions with group dialogical methods and Owela were utilised. The Living Lab community seemingly benefited from the social agenda and the technical platforms set by the Living Lab, as the participants were encouraged to meet each other and collaborate for the common goals of the Living Lab.

\subsection{Responsibility}

HDD goes beyond the design of products and systems to the wellbeing of people and sustainability. In this sense, HDD can be part of the emerging activity of responsible innovation (RRI, Responsible Research and Innovation). RRI refers to "a transparent, interactive process by which societal actors and innovators become mutually responsive to each other with a view on the (ethical) acceptability, sustainability and societal desirability of the innovation process and its marketable products" [28]. We suggest by design thinking here, in order to promote responsibility in design outcomes, e.g. "Ethics by design" is a model to integrate consideration of ethical issues deeply into the technology development process from the early phase so that ethics is not an external part of a development project but rather an ongoing dialogue throughout the development span [29].

Ethics by Design. During the last decade, we have participated as HCI/HTI researchers in research focusing on the ethics of emerging ICTs and, for instance, developed ethical guidelines for mobile-centric ambient intelligence [30]. With regard to emerging ICTs, we have noticed a need for thinking and practices aimed at a positive ethical contribution in system design [cf. 29]. Ethical principles and questions should work as sources of innovation for designing ethically sound concepts. This thinking can be called ethics by design, extending from Marc van Lieshout and colleagues' model of privacy by design [31].

In ethics by design, ethical considerations are involved from the very beginning of the development process and embedded through the process. All stakeholders including technology developers, direct users and indirect users are involved in ethical deliberation through representatives, e.g. in focus groups. In addition, to ensure sufficiently deep understanding of ethical issues, ethical experts can work for the project, either as internal project workers or as an external expert board. Ethics is also included at the user end so that the user experience of ethicality of design outcomes is considered.

Sustainability by Design. Here, sustainability is defined with regard to sustainable development "that meets the needs of the present without compromising the ability of future generations to meet their own needs" [32]. A common view is to consider sustainability along three dimensions that focus on environmental, social and economic long-term consequences. 
Within HCI, research related to environmental sustainability, in particular, has expanded in recent years. In 2007, Jennifer Mankoff and colleagues introduced several pathways for HCI to contribute to the well-being of the environment [33]. Once again, we have paid special attention to the sustainability by (through) design perspective. This view considers ways to support sustainable lifestyles and decision-making through the design of technology. Sustainability by design sees technology as a "channel for intervention in the everyday decisions and mindsets" [33] that, partly, underlies environmental problems. In line with this, we have developed a visual, online co-design tool to provide easy ways to co-create and share meanings in regard to physical environments and, in this way, encourage shifting the users' practices toward sustainability (see Section 3.2 and [27]).

\section{Discussion}

This paper presents an attempt to collect and elaborate on principles for a humandriven approach to technology and interaction design. Based on the well-known frameworks of design such as Human-Centred Design, Participatory Design and Value Sensitive Design, Human-Driven Design takes human and social values profoundly into account and aims to take real human needs as a starting point of the design. HDD endorses participation and empowering of users and other stakeholders by developing and applying co-design methods, of which we have provided examples. The general aim of HDD should be to involve people in the design of emerging technologies and human-technology interaction concepts so that their needs and values become articulated in the design process. At the same time, the process should include reflection on ethical and sustainability terms. HDD also promotes responsible research and innovation, e.g., in the forms of ethics by design and sustainability by design. In fact, HDD can be seen as an attempt to take the framework of responsible research and innovation (e.g. [28]) in practice.

We have developed HDD thinking in the context of designing emerging technologies, that is, technologies that are currently being developed and that hold realistic potential to become widely adopted within the next 10 to 20 years [34]. This includes concepts like ambient intelligence, ubiquitous computing, autonomous systems, emotional computing, service robotics and convergence of ICT with other technologies, e.g. nanotechnology or human implants. In addition to the human and social view, participation and responsibility, HDD should try to understand the future contexts in which certain technology would be embedded. Arguably, HCI has capitalised very little on systematic methods of futures thinking, although this could "prepare us for problems that have not yet arrived, enable the 'invention' of a better future, or increase the long-term impact of our work" [35, p. 1629]. Our next step is to study relevant methods to gain an insight into the future, especially in terms of human and social behaviour and societal trends, i.e. to study human-driven foresight. This step is necessary for us to be able to formulate HDD as a useful alternative design approach to emerging ICTs that is deeply based on respecting human values and needs as well as sustainability. 
Acknowledgements. We would like to thank the reviewers for their helpful comments. This work has been funded by the GREAT project (EU FP7/grant agreement no. 321480) and supported by the VTT Design for Life innovation program.

\section{References}

1. Bannon, L.: Reimagining HCI: toward a more human-centered perspective. Interactions 18(4), 50-57 (2011)

2. Friedland, B., Yamauchi, Y.: Reflexive design thinking: putting more human in humancentered practices. Interactions 18(2), 66-71 (2011)

3. Steen, M.: The fragility of human-centred design. Doctoral Thesis, Delft University of Technology (2008)

4. Braund, P., Schwittay, A.: The Missing Piece: Human Driven Design and Research in ICT and Development. In: Proceedings of the International Conference on Information and Communications Technologies and Development, May 25-26, pp. 2-10 (2006)

5. Ikonen, V.: Towards empowering design practices. In: Proceedings of the Ambient Intelligence Forum 2008, Hradec Králové, CZ, October 15-16, pp. 132-139 (2008)

6. Norros, L., Kaasinen, E., Plomp, J., Rämä, P.: Human-Technology Interaction Research and Design. VTT Roadmap, VTT Industrial Systems, Espoo (2003)

7. Leikas, J.: Life-Based Design - A holistic approach to designing human-technology interaction. VTT Publications 726, Edita Prima Oy, Helsinki (2009), http: / /www.vtt.fi/inf/pdf/publications/2009/P726.pdf

8. ISO: Ergonomics of human-system interaction. Part 210: Human-centred design for interactive systems, ISO 9241-210:2010 (2010)

9. Bohn, J., Coroama, V., Langheinrich, M., Mattern, F., Rohs, M.: Living in a World of Smart Everyday Objects - Social, Economic, and Ethical Implications. Journal of Human and Ecological Risk Assessment 10(5), 763-786 (2004)

10. Greenbaum, J., Kyng, M.: Design at work: Cooperative design of computer systems. CRC (1991)

11. Holone, H., Herstad, J.: Three Tensions in Participatory Design for Inclusion. In: Proceedings of the SIGCHI Conference on Human Factors in Computing Systems, Paris, France, pp. 2903-2906. ACM, New York (2013)

12. Sanders, E.B.-N., Stappers, P.J.: Co-creation and the new landscapes of design. CoDesign: International Journal of CoCreation in Design and the Arts 4(1), 5-18 (2008)

13. Friedman, B., Kahn Jr., P.H.: Human values, ethics, and design. In: Jacko, J.A., Sears, A. (eds.) The Human-Computer Interaction Handbook, pp. 1177-1201. Lawrence Erlbaum Associates, Mahwah (2003)

14. Friedman, B., Kahn, P.H.: Jr., Borning, A.: Value Sensitive Design and information systems. In: Zhang, P., Galletta, D. (eds.) Human-Computer Interaction in Management Information Systems: Foundations, pp. 348-372. M.E. Sharpe, London (2006)

15. Borning, A., Muller, M.: Next steps for value sensitive design. In: Proceedings of the SIGCHI Conference on Human Factors in Computing Systems, pp. 1125-1134. ACM, New York (2013)

16. Huldtgren, A.: Addendum: Practical Considerations of Value Sensitive Design. In: Doorn, N., Schuurbiers, D., van de Poel, I., Gorman, M.E. (eds.) Early Engagement and New Technologies: Opening Up the Laboratory, pp. 85-95. Springer, Dordrecht (2013) 
17. Manders-Huits, N., Zimmer, M.: Values and Pragmatic Action: The Challenges of Introducing Ethical Intelligence in Technical Design Communities. International Review of Information Ethics 10, 37-44 (2009)

18. Leikas, J., Saariluoma, P., Rousi, R., Kuisma, E., Vilpponen, H.: Life-based design to combat loneliness among older people. Journal of Community Informatics 8, 1 (2012), http://ci-journal.net/index.php/ciej/article/view/778/890

19. Leikas, J., Saariluoma, P., Heinilä, J., Ylikauppila, M.: A Methodological Model for LifeBased Design. International Review of Social Sciences and Humanities (IRSSH) 4(2), 118-136 (2013), http: / / irssh.com/yahoo_site_admin/assets / docs/11_IRSSH-415-V4N2 .44203734.pdf

20. Saariluoma, P., Leikas, J.: Life-Based Design - an approach to design for life. Global Journal of Management and Business Research (GJMBR) 10(5), 17-23 (2010), http: //journalofbusiness .org/index.php/GJMBR/article/view/ $199 / 174$

21. Datye, S.: Life-Based Design for Technical Solutions in Social and Voluntary Work. Jyväskylä University Printing House, Jyväskylä (2012)

22. Pinkett, R.: Bridging the Digital Divide: Sociocultural Constructionism and an AssetBased Approach to Community Technology and Community Building. In: 81st Annual Meeting of the American Educational Research Association (2000)

23. Kaasinen, E., Koskela-Huotari, K., Ikonen, V., Niemelä, M., Näkki, P.: Three approaches to co-creating services with users. In: Proceedings 4th International Conference on Applied Human Factors and Ergonomics, San Francisco, USA, July 21-25 (2012)

24. Ikonen, V., Hakulinen, J., Kivinen, T., Aloja, M., Hokkanen, L., Ruutikainen, P., Kymäläinen, T., Nelimarkka, M., Harrela, A.: IHME = Miracle - Make, Imagine and Research Applications for Computerised Living Environments. In: Proceedings of the 14th International Academic MindTrek Conference: Envisioning Future Media Environments, Tampere, Finland, pp. 3-6 (2010)

25. Näkki, P., Antikainen, M.: Online tools for co-design: user involvement through the innovation process. In: Karahasanovic, A., Følstad, A. (eds.) The NordiCHI 2008 Workshops: New Approaches to Requirements Elicitation \& How Can HCI Improve Social Media Development? Tapir Akademiskforlag, Trondheim, pp. 92-97 (2008)

26. Niemelä, M., Kivinen, T., Kulju, M., Tammela, A., Ikonen, V., Korhonen, H.: Visual IHME: co-designing meaningful places for sustainability. In: IADIS Interfaces and $\mathrm{Hu}-$ man-Computer Interaction Conference, Czech Republic, July 22-24 (2013)

27. Niemelä, M., Kivinen, T., Kulju, M., Tammela, A., Ikonen, V., Korhonen, H.: Visual IHME: co-designing meaningful places for sustainability. In: Isaías, P., Blashki, K. (eds.) Human-Computer Interfaces and Interactivity: Emergent Research and Applications. IGI Global (in press)

28. Von Schomberg, R. (ed.): Towards Responsible Research and Innovation in the Information and Communication Technologies and Security Technologies Fields. Publication Office of the European Union, Luxembourg (2011), http: / / ec . europa . eu / research/science-society/document_library/pdf_06/ mep-rapport-2011_en.pdf

29. Guardian Angels: FET Flagship pilot, final report (July 2012), public version (2012), http://www.ga-project.eu/files/content/sites/ guardians-angels-neutre/files/pdf/Guardian_Angels_ Final_Report_July_2012.pdf 
30. Ikonen, V., Kaasinen, E., Niemelä, M.: Defining Ethical Guidelines for Ambient Intelligence Applications on a Mobile Phone. In: Workshops Proceedings of the 5th International Conference on Intelligent Environments, Barcelona, ES, pp. 261-268. IOS Press, Amsterdam (2009)

31. van Lieshout, M., Kool, L., van Schoonhoven, B., de Jonge, M.: Privacy by Design: an alternative to existing practice in safeguarding privacy. Info 13(6), 55-68 (2011)

32. World Commission on Environment and Development: Our Common Future (Brundtland Report). Oxford University Press, Oxford (1987)

33. Mankoff, J., Blevis, E., Borning, A., Friedman, B., Fussell, S.R., Hasbrouck, J., Sengers, P., Woodroff, A.: Environmental sustainability and interaction. In: Proceedings of the SIGCHI Conference on Human Factors in Computing Systems, San Jose, CA, USA, pp. 2121-2124. ACM, New York (2007)

34. Stahl, B.C., Heersmink, R., Goujon, P., Flick, C., van de Hoven, J., Wakunuma, K., Ikonen, V., Rader, M.: Issues, Concepts and Methods Relating to the Identification of the Ethics of Emerging ICTs. Communications of the IIMA 10(1) (2010)

35. Mankoff, J., Rode, J.A., Faste, H.: Looking past yesterday's tomorrow: using future studies methods to extend the research horizon. In: Proceedings of the SIGCHI Conference on Human Factors in Computing Systems, Paris, France, pp. 1629-1638. ACM, New York (2013) 